\title{
Harnessing the flexibility of energy management systems: a retailer perspective
}

\author{
Sébastien Mathieu*, Miguel Manuel de Villena*, Eric Vermeulen ${ }^{\dagger}$, and Damien Ernst* \\ ${ }^{*}$ University of Liege, Liege, Belgium \\ Email: smathieu, mvillena, dernst \{@uliege.be \\ †haulogy, Gosselies, Belgium \\ Email: eric.vermeulen@haulogy.net
}

\begin{abstract}
The business of electricity retailing is changing following the current evolution of the electricity system. An example of such an evolution is the increasing number of clients installing sophisticated energy management systems controlling the production or consumption of a large variety of devices as, for instance, photovoltaic panels with storage solutions. These systems, if managed properly, may provide flexibility to the electricity system to which they are connected, typically by means of an intermediary such as a retailer. Retailers could transfer such flexibility in consumption/production of electricity to the day-ahead and intraday electricity markets, trade it with other balance responsible parties, or use it to participate in the imbalance market. This paper defines an interaction model between a retailer and its clients, based on generic flexibility bids over multiple market periods. The defined interaction model relies on smart meters for registering the trades of electricity and requires no modification of the current rules and regulations of the European electricity system. The concept of flexibility is defined with respect to a baseline, which is negotiated and agreed upon between the retailer and each client. This baseline can be modified by the client following an update mechanism, which must comply with certain security checks by the retailer in order to make sure it is not fraudulent. Any deviations from the quarter-hourly comparison between expected and actual production/consumption are invoiced at a discounted price.
\end{abstract}

\section{INTRODUCTION}

The role of a traditional electricity retailer comprises acting as an intermediary between its clients and the rest of the electricity system. In its role, a retailer purchases electricity for its clients based on consumption forecasts, acts as balance responsible party (BRP) for the transmission system operator (TSO), and invoices its clients. The interaction between the retailer and its clients works one way: the client benefits from the system but does not contribute to it. To be able to manage this interaction efficiently, an increasing number of clients are installing (or upgrading) sophisticated energy management systems (EMS) to save energy in a cost-efficient manner [1]. An EMS is an automated energy controller using a computer as a central processor. The capabilities of the EMS may vary widely depending on the selected model. Nonetheless, its basic capabilities are almost universal, and notably comprise the scheduling of the electricity flows, fixing the set-points of the batteries, alarms and safety measurements, and basic system monitoring.

An EMS can manage the production or consumption of a large variety of devices, such as photovoltaic panels, storage solutions (e.g. batteries), and flexible loads (e.g. demandflexible boilers). The nature of the constraints of these different devices thus require dedicated management, for instance, one EMS may be designed to control the appliances of a house, as described in [2], whilst another one could be designed to control a microgrid with several companies, photovoltaic panels, a run-on-the-river generator, and a storage system, as detailed in [3]. Furthermore, there might be cases where an EMS simply controls demand response devices. From the standpoint of a retailer, an EMS represents a higher-level manager of all these devices (i.e. generation, storage, or flexible demand) to provide flexibility. The flexibility thus provided by the clients' EMSs is then forwarded by the retailer to the day-ahead and intraday electricity markets. Alternatively, it can be exchanged with other BRPs, or be used by the retailer to participate in the imbalance market. In this regard, the retailer can be interpreted as a smart BRP providing single generic access to various flexibility products, aggregating this flexibility to meet minimum volume constraints, simplifying accounting, and managing the flexibility of its clients. This paper aims at defining an interaction model between a retailer and several clients (through the clients' EMSs) to exchange flexibility. The interactions are based on a generic interface with the EMS, ensuring the scalability of the method, which should be simple for the most basic EMS, yet able to include the constraints of the controlled devices and, in particular, rebound effects [4]. In this context, flexible trading should be an addition to traditional electricity retailing. If no flexibility is traded, the contract between the client and the retailer corresponds to a classic retailing contract. The scientific literature covers most of the components required to define such an interaction model. However, to date, there is no implementation covering all the requirements together.

For the remainder of this paper: the next section reviews the relevant literature. This review is followed by an outline of the proposed interaction model. Specific components of the model are detailed in dedicated sections: the baseline and its update, the flexibility bids, and the deviation mechanism. Finally, the last section concludes the paper and identifies the potential future prospective work. 


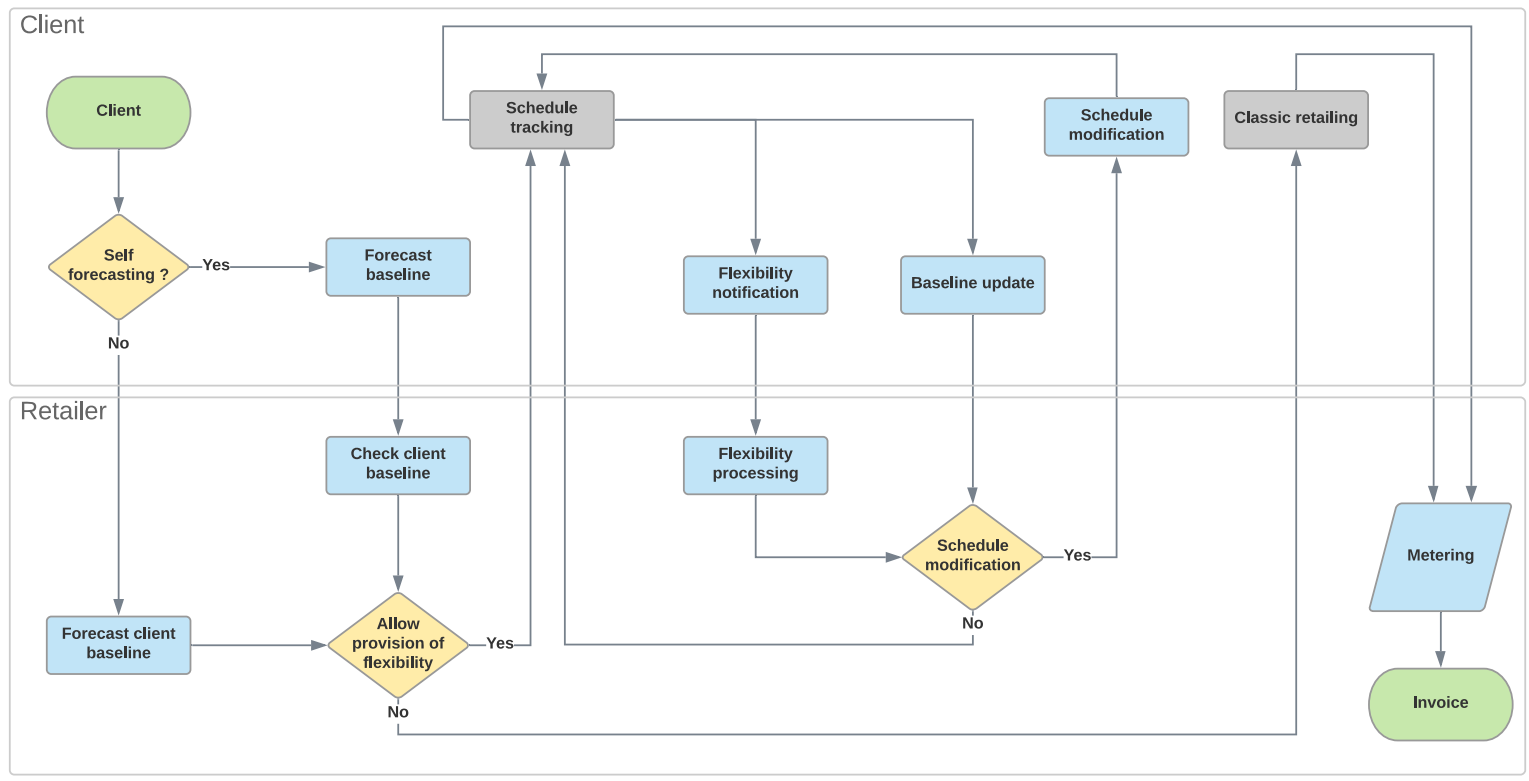

Fig. 1: Flow of interactions between a client and its retailer. A baseline is computed for each client. Then the retailer allows, or not, the provision of flexibility of the client. If it is not accepted, the client falls under a classic retailing contract. If accepted, the client notifies its capability to provide flexibility. If the retailer contracts the flexibility, the schedule of the client is modified accordingly. This schedule may be modified upon notification of the client. The client is invoiced based on the final schedule and the metered energy.

\section{LITERATURE REVIEW}

The existing literature regarding the use of demand response to provide flexibility is abundant, see for instance reviews [5] and [6]. The latter was conducted in 2018 and presents the results of 60 works [6]. Furthermore, many projects on this topic have been conducted over recent years. ADDRESS started in 2008 and is one of the earliest European projects dedicated to demand response [7]. BRIDGE is a European Commission initiative that unites Horizon 2020 Smart Grid and Energy Storage Projects [8]. The BRIDGE project recommends implementing the Winter Package directives into the market system regulation based on dedicated recommendations related to specific dimensions: demand response access to markets, service providers' access to markets, product requirements, measurement and verification, payments, and penalties [8]. To enable flexibility services, the authors in [7] highlight the importance of introducing minor modifications in existing markets, rather than creating new markets. In this process, the retailer is well-placed to act as a facilitator.

The interaction model proposed in this paper is a marketbased approach. Other examples of such approaches for flexibility aggregation can be found in the Flexiciency [9] and PowerMatcher [10] projects. The former details a European market place facilitating interaction between agents with advanced monitoring, local energy control, and flexibility of aggregated customers. Flexibility services are very generic and must define various parameters such as the payment model, preconditions for service, or detailed description of service delivery. Concerning PowerMatcher, this project represents a practical implementation of market-based aggregation; it operates as a smart grid coordination mechanism balancing distributed energy resources (such as renewable ones) and flexible demand. First, different devices send bids detailing their willingness to consume energy, and then the aggregator sends back a price signal so that they can determine its consumption volume at this price.

The mentioned projects interact with various types of devices, either using generic but complex models, or several specific ones. The authors in [6] claim that, to create a wellfunctioning interaction model, it is necessary to formulate standardized but simple definitions of flexibility products, accounting for energy-constrained resources, flexibility capacity shortage situations, and including the rebound effect. One step toward the definition of such flexibility products is proposed in [11], where a solution is tailored to harness the flexibility from heat pumps. The flexibility product introduced in the latter work is used as the base block of the interaction model proposed in this paper.

\section{Proposed INTERACTION MODEL}

The proposed interaction model between a client willing to provide flexibility and its retailer complies with the following outline: a client provides a baseline, based on its own consumption forecasts and additional forecasts if needed. If the EMS of the client has no forecasting capabilities, a reference is built from historical values. The retailer accepts or declines the participation of the client in its flexibility pool. Once accepted, the EMS of the client computes its capability to provide flexibility and communicates it to the retailer. 
The retailer processes all flexibility offers coming from the different clients' EMSs, taking into account the current status of the energy markets and its requirements as a BRP. If the retailer contracts flexibility, the schedule of the relevant clients are modified accordingly. Simultaneously, forecast updates may be requested by the client. The retailer checks if the new baseline is valid and does not impair the provision of previously accepted flexibility. Finally, the client is invoiced based on the final schedule and the metered energy. Deviations from the reference, with a specified tolerance, are penalized by the retailer. The flow of interactions is illustrated in Figure 1. This interaction model relies on smart metering and requires no modification of the current electricity market.

\section{BASELINE AND UPDATES}

A baseline is necessary to define the flexibility provided at a resolution of a minimum 15 minutes, for its use in most electricity markets. Such a baseline is a specific requirement of this kind of client-retailer interaction. For classic retailing contracts, the baseline of a client is not compulsory. The retailer assumes the role of BRP for the clients it represents. In this setting, the TSO computes the potential imbalance of the retailer with respect to its net position. The computation of the net position of the retailer is based on its electricity purchases, which are in turn based on the forecasts of its clients. Large consumers may be requested to provide baselines to the retailer. In that instance, a communication of the baseline will be imposed by the retailer's contract (i.e. as an agreement between the client and the retailer). Hence, the TSO may not be aware of the baseline of the client and only has information concerning the schedule of the retailer's portfolio.

If flexibility is sold to the TSO, the baseline may be defined by the TSO itself [12], [13]. Taking another reference would create a mismatch between the flexibility remunerated by the TSO and the flexibility provided by the client. In this case, the retailer communicates to the client the reference taken by the TSO, or the method if the necessary data are not available in advance.

If flexibility is sold only as a result of a change in the retailer's net position, the definition of the client baseline is only an agreement between the client and the retailer. Since it is technically challenging to predict the state of an EMS without the details of the underlying devices, the EMS should provide its planned schedule to the retailer. According to [14], baseline and flexibility should be computed by the EMS to ensure end-user privacy and comfort. However, a concern regarding the self-computation of the baseline and flexibility is that customers might attempt to purposely manipulate their baseline in order to maximize their profits. The typical way of "gaming" the baseline is that customers may declare a higher consumption than their needs during their peak demand to sell flexibility in the form of a fictitious reduction of consumption. However, such abuses may not be intentional. Any optimization-based controller naturally exploits the flaws of a deficient interaction model. To prevent these problems, a retailer can compare the information provided by the client with its own forecasts. This check is essential to detect anomalies and can be used to avoid abuses of the flexibility mechanism. One method to prevent such potential abuses is to check the similarity of the communicated baseline with historical measurements, applying a tolerance.

The baseline must not only cover the period in which the client is willing to provide flexibility, but also some periods before and after flexibility delivery, to consider the rebound effect. The length of these periods depends, among other factors, on the storage capacity behind the EMS controller. The order of magnitude of residential thermal storage, for instance, is one and a half hours [11], whereas exploring the opposite extreme, a microgrid storage system may shift consumption by several hours. Considering these orders of magnitude, a baseline window of one day around a flexibility window is considered for the present paper, for this is assumed to be a reasonable length.

A baseline should be defined before the clearing of the dayahead energy market so that the retailer has sufficient time to compute and issue its offers. This baseline should therefore be computed based on day-ahead forecasts. Typically, more accurate forecasts can be obtained closer to real-time, leading to the need for baseline updates. However, an update may not always be accepted, since it could compensate for previously sold flexibility or an unspecified rebound effect. Thus, two verification points are suggested: (i) a maximum relative deviation with respect to the initial baseline, and (ii) prevention of baseline modification in the opposite direction to the provision of flexibility services. Figure 2 shows a schedule update which cancels out already sold flexibility: (a) assuming a client with a flat baseline; (b) the client sells flexibility and the schedule is modified accordingly; (c) the client requests a schedule update in the opposite direction to its sold flexibility. If this update is accepted, the client is paid for a flexibility it does provide. To avoid such potential abuses, the retailer should not accept a baseline update of an opposing sign than the sold flexibility. In practice, a small tolerance corresponding to acceptable forecast errors should be considered.

\section{FleXibility BIDS}

A client communicates its flexibility by means of bids. This flexibility product is inspired by proposals of articles [11] and [15]. It consists of an offer covering multiple market periods in which signs may vary. This product generalizes the case of a single period offer. A bid communicates the flexibility over multiple time-steps and includes the following information:

- Energy volume for each time step;

- Type: partial/binary acceptance;

- Cost of the bid; and

- Expiration time.

A graphical representation of such a bid is provided in Figure 3. The retailer selects interesting bids, either to directly use them, or to be sold (aggregated or not) to other BRPs or to the intraday market. As for the market clearing process, many implementations can be investigated. In any case, the clearing procedure should be adequate to exchange flexibility close to 

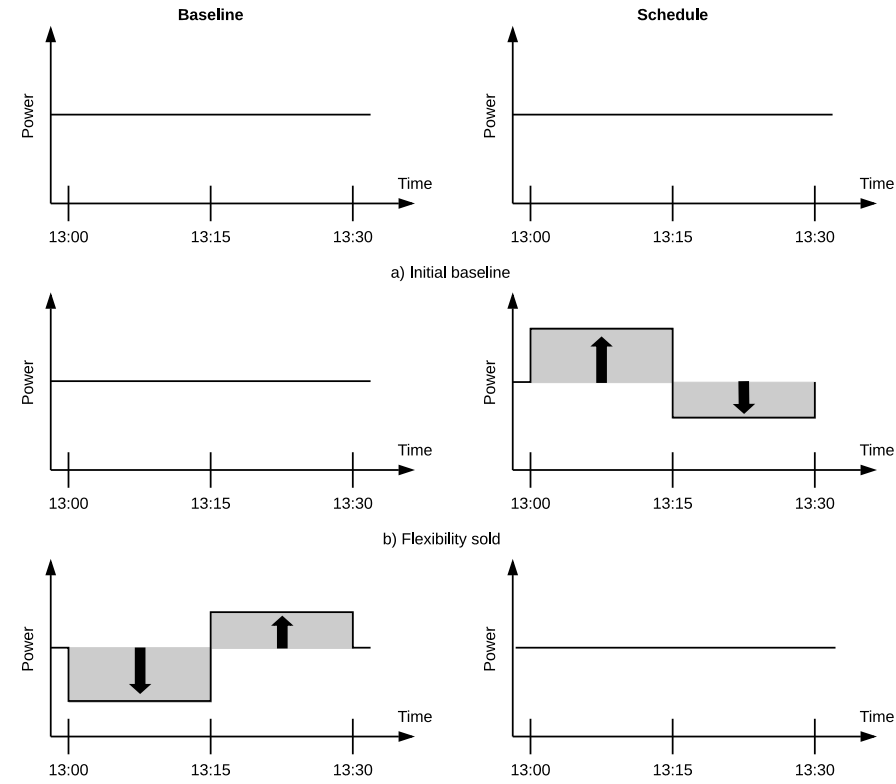

c) Baseline update

Fig. 2: Case of a schedule update that cancels out already sold flexibility.

real-time as, for example, in the real-time balancing settlement. The latter encourages the use of continuous clearings.
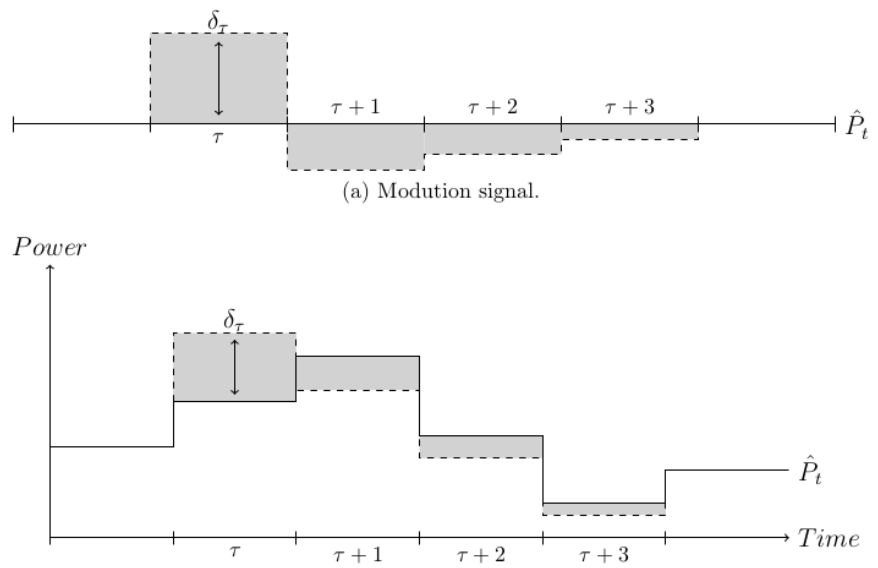

(b) Modulation added to the baseline.

Fig. 3: Modulation of the consumption offered as a flexibility bid [11].

By default, a bid allows partial acceptance of the offered flexibility volumes. The accepted flexibility volume at each time-step is given by the offered volume multiplied by the acceptance ratio. The client can prevent the possibility of partial acceptance for any bid, imposing the complete acceptance or rejection condition, namely, using the binary bids.

The response of the retailer to the bid submission is the status of the bid, which can take the following values:

- FREE: Bid submitted and free to be revoked by the client.

- REVOKED: Bid revoked by the client.
- PENDING: The retailer is processing the flexibility bid and it cannot be revoked.

- EXPIRED: The bid reached its expiration time without being accepted or rejected.

- REJECTED: Bid rejected by the retailer.

- RESERVED: The bid is reserved and waits for its acceptance by its future beneficiary.

- ACCEPTED: The bid is accepted and an acceptance ratio is communicated.

Figure 4 illustrates the evolution of bid statuses. Clients submit their offers to the retailers, which are initially in a free state. They are always free to revoke an offer if it has not been processed. Periodically, the retailer processes the current free offers. During the processing phase, the statuses of the concerned offers is set as pending. The retailer first filters offers that have expired due to a time-out. Next, it selects offers to be submitted to the markets or proposed to the TSO or other BRPs. These offers are then set to a reserved status, waiting for acceptance, while the rest of them are switched back to the free status. The retailer could implement a wide range of strategies to process bids. A basic one would be to forward the bids directly to the markets, in order of profitability, discarding the ones not suitable for participation (e.g. the ones which will not generate profits). A more advanced strategy could consist of building a market offer by aggregating a collection of flexibility bids, however, the definition of this kind of algorithm is out of the scope of this document. The algorithm proposed in this paper is assumed to send offers to the market, communicating back to the retailer the provided flexibility. The retailer then dispatches this flexibility to the reserved offers and sets their status as accepted. Concerning the rest of the offers, they are switched back to the free status for the next selection process.

Once a flexibility bid is accepted, the schedule of the corresponding client is modified accordingly. Note that following this principle, a client has an alternative to the baseline update mechanism to modify its schedule. The client could bid the expected schedule update at an appealing price with respect to the one of the intraday market. Thus, the retailer could buy or sell the corresponding energy on the market and update the schedule of the client accordingly.

\section{DEVIATION MECHANISM}

A deviation is given by the difference between the measured consumption and the baseline (i.e. the foreseen consumption plus the provided flexibility). Nonetheless, these deviations are not considered an imbalance in this document since the clients are not BRPs.

The retailer benefits from averaging its portfolio of clients to mitigate the variability of its forecasts and deviations. The client cannot benefit from this averaging effect owing to its small size and limited flexibility. Before pricing a deviation, a retailer may therefore grant a tolerance to its clients for deviations with respect to their schedules. Beyond this tolerance, a price needs to be associated with this deviation. The imbalance price is a good candidate since it corresponds to the cost faced 


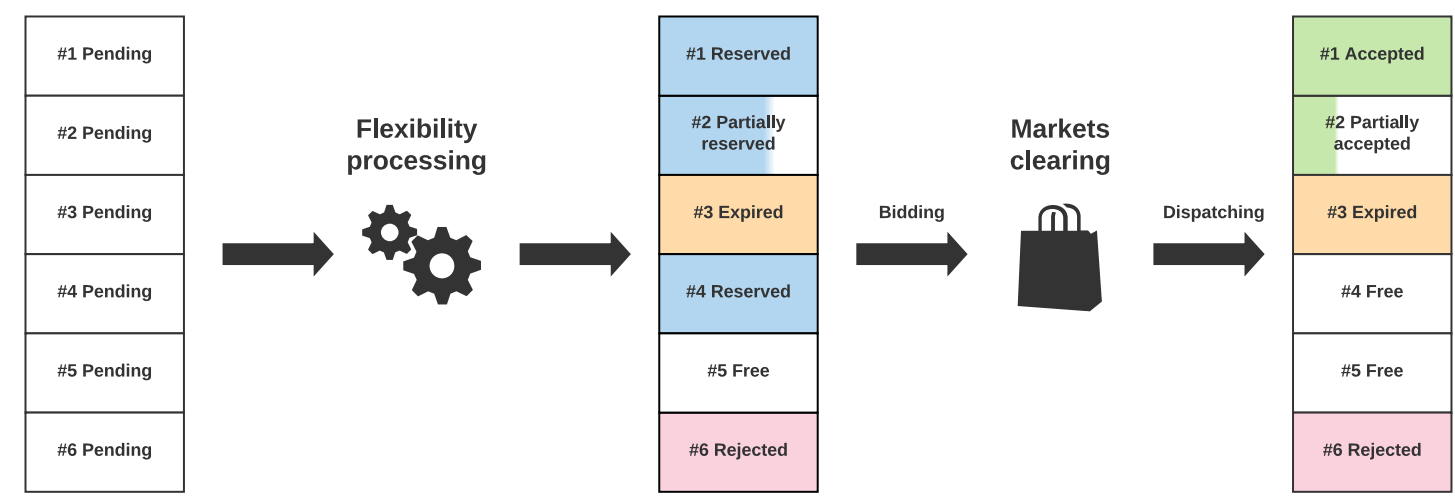

Fig. 4: Evolution of flexibility bid statuses.

by the retailer. Furthermore, exposing the client to imbalance prices might encourage them to enroll in a flexibility program. The retailer could reduce the deviation price by a factor corresponding to the reduction of the risk resulting from the aggregation of the clients. This reduction can be computed as follows. Considering a retailer with $k$ identical clients with forecast errors following Gaussian distributions correlated by a factor $\rho$. The production of the $i^{t h}$ client is given by $p_{i, t}$, whereas its production forecast is $\hat{p}_{i, t}$. Then, the covariance of a pair of clients $i, j$ is given by $\rho \sigma_{i} \sigma_{j}$. The sum of correlated Gaussian random variables is studied in paper [16]. In this paper, the authors prove that the sum of correlated normally distributed random variables is equal to one single random variable following a normal distribution of variance $\sigma_{Z}=\sigma_{i}^{2} \sum_{i=1}^{k} \sum_{j=1}^{k} \rho_{i, j}$ where $\rho_{i, j}$ is the covariance.

Using this finding, the aggregated forecast error is given by the sum of the individual distributions. Let $P_{t}$ and $\hat{P}_{t}$ denote the production and the estimated production of the retailer, which corresponds to the sum of the production and estimated production of its clients.

$$
\begin{aligned}
E\left[P_{t}-\hat{P}_{t}\right] & =N\left(0, \sigma_{i}^{2} \sum_{i=1}^{k} \sum_{j=1}^{k} \rho_{i, j}\right) \\
& =N\left(0, \sigma_{i}^{2} \sum_{i=1}^{k}(1+(k-1) \rho)\right) \\
& =N\left(0, \sigma_{i}^{2} k(1+(k-1) \rho)\right)
\end{aligned}
$$

The relative standard deviation of the error $C$ is given by:

$$
C=\frac{E\left[P_{t}-\hat{P}_{t}\right]}{E\left[P_{t}\right]}=\frac{\sqrt{k+k(k-1) \rho}}{k} c_{i}
$$

where $c_{i}$ is the relative standard deviation of the client's error. We can define the factor $\phi$, representing the influence of the correlation of the client's production on the total production of the retailer as:

$$
\phi=\frac{C}{c_{i}}=\frac{\sqrt{k+k(k-1) \rho}}{k}
$$

The computation of $\phi$ for different numbers of clients $k$ and for different correlation coefficients $\rho$ is showcased in Table
TABLE I: Influence of the correlation of the clients' production on the total production of the retailer, as a function of the number of clients $k$ and the correlation of their production $\rho$.

\begin{tabular}{lrrrrrr}
$k / \rho$ & $\mathbf{0}$ & $\mathbf{0 . 1}$ & $\mathbf{0 . 2}$ & \multicolumn{1}{c}{$\mathbf{0 . 3}$} & $\mathbf{0 . 4}$ & \multicolumn{1}{c}{$\mathbf{0 . 5}$} \\
\hline $\mathbf{1}$ & $100 \%$ & $100 \%$ & $100 \%$ & $100 \%$ & $100 \%$ & $100 \%$ \\
$\mathbf{2}$ & $71 \%$ & $74 \%$ & $77 \%$ & $81 \%$ & $84 \%$ & $87 \%$ \\
$\mathbf{3}$ & $58 \%$ & $63 \%$ & $68 \%$ & $73 \%$ & $77 \%$ & $82 \%$ \\
$\mathbf{5}$ & $45 \%$ & $53 \%$ & $60 \%$ & $66 \%$ & $72 \%$ & $77 \%$ \\
$\mathbf{1 0}$ & $32 \%$ & $44 \%$ & $53 \%$ & $61 \%$ & $68 \%$ & $74 \%$ \\
$\mathbf{1 0 0}$ & $10 \%$ & $33 \%$ & $46 \%$ & $55 \%$ & $64 \%$ & $71 \%$ \\
$\mathbf{1 0 0 0}$ & $3 \%$ & $32 \%$ & $45 \%$ & $55 \%$ & $63 \%$ & $71 \%$ \\
$\mathbf{1 0 0 0 0}$ & $1 \%$ & $32 \%$ & $45 \%$ & $55 \%$ & $63 \%$ & $71 \%$
\end{tabular}

I. This table shows that for a sufficient number of clients, with a realistic correlation of 0.2 , the relative forecast error of the retailer is $45 \%$ of the one an individual client would obtain. Thus, $45 \%$ could be used as a potential discount on the imbalance price to define the deviation price of the client.

\section{CONCLUSION}

This paper presents an interaction model allowing a set of clients equipped EMSs to provide flexibility services to the electrical system through their retailer. The scope of this interaction model covers energy exchanges spanning from the day-ahead to real-time. These exchanges may be simple for the most basic EMSs, while allowing clients with device constraints such as rebound effects to include them in such exchanges. The trading of flexibility is an addition to traditional retailing. If no flexibility is traded, the contract between the client and the retailer corresponds to a classical one.

This interaction model could be extended to capacity exchanges by defining capacity products. The capacity would be activated by the retailer depending on the needs. In this case, an offer should include a reservation and an activation cost. Another alternative flexibility market is one where the retailer notifies all EMSs of a flexibility need. The EMSs then respond with their flexibility offers which may be processed as described in this paper.

\section{ACKNOWLEDGMENT}

This research is supported by the public service of Wallonia (DGO6) within the framework of the Haulogy 2021 project. 


\section{REFERENCES}

[1] K Stum, R Mosier, and T Haasl, "Energy management systems-a practical guide," Portland Energy Conservation Inc.(PECI), USA, p. 116, 1997.

[2] R. Missaoui, H. Joumaa, S. Ploix, and S. Bacha, "Managing energy smart homes according to energy prices: Analysis of a building energy management system," Energy and Buildings, vol. 71, pp. 155-167, 2014.

[3] B. Cornélusse, D. Ernst, L. Warichet, and W. Legros, "Efficient management of a connected microgrid in belgium," CIRED-Open Access Proceedings Journal, vol. 2017, no. 1, pp. 1729-1732, 2017.

[4] S. Mathieu, "Flexibility services in the electrical system," PhD thesis, University of Liège, Belgium, 2016.

[5] R. J. Bessa and M. A. Matos, "Economic and technical management of an aggregation agent for electric vehicles: A literature survey," European transactions on electrical power, vol. 22, no. 3, pp. 334-350, 2012.

[6] J. Villar, R. Bessa, and M. Matos, "Flexibility products and markets: Literature review," Electric Power Systems Research, vol. 154, pp. 329-340, 2018.

[7] A Losi, P Mancarella, S Mander, G Valtorta, P Linares, A Horch, et al., "ADDRESS recommendations for standard committees, regulators, stakeholders groups, future R\&D," Brussels, Belgium, 2013.
[8] B. H. 2020, Bridge business models working group, second report: Business models issues, Apr. 2018. [Online]. Available: https://www.h2020-bridge.eu.

[9] FLEXICIENCY, Deliverable d2.1: Definition of services and use cases description. Jan. 2016.

[10] J. Kok, "The powermatcher: Smart coordination for the smart electricity grid," 2013.

[11] E. Georges, B. Cornélusse, D. Ernst, V. Lemort, and S. Mathieu, "Residential heat pump as flexible load for direct control service with parametrized duration and rebound effect," Applied Energy, vol. 187, pp. 140-153, 2017.

[12] RTE, Règles pour la valorisation des effacements de consommation sur les marchés de l'énergie NEBEF 3.1, 2018.

[13] ELIA, Règles organisant le transfert d'énergie. 2018.

[14] M. L. D. Heleno, "Provision of advanced ancillary services through demand side integration," 2016.

[15] J. A. Schachter and P. Mancarella, "Demand response contracts as real options: A probabilistic evaluation framework under short-term and long-term uncertainties," IEEE Transactions on Smart Grid, vol. 7, no. 2, pp. 868-878, 2016.

[16] J. Fowler, Density function for the sum of correlated random variables, 2011. 